\title{
THE BIRKHOFF ORTHOGONALITY IN PRE-HILBERT $C^{*}$-MODULES
}

\author{
PAWEŁ WÓJCIK
}

Abstract. In this work we characterize the Birkhoff orthogonality for elements and finite dimensional subspaces of a pre-Hilbert $C^{*}$-module in terms of a convex hull of continuous linear functionals. The aim of the paper is to present results concerning the $B$-orthogonality and its applications. We also present the results concerning smoothness. Moreover, we give a new proof of the Bhatia-Šemrl theorem.

Mathematics subject classification (2010): 46B20, 46L08, 46L05.

Keywords and phrases: Pre-Hilbert $C^{*}$-module, continuous linear functional, smoothness.

\section{REFERENCES}

[1] LJ. ARAMBAŠIĆ AND R. RAJIĆ, The Birkhoff-James orthogonality in Hilbert $C^{*}$ - modules, Linear Algebra Appl. 437 (2012), no. 7, 1913-1929.

[2] R. BhatiA, P. ŠEMRL, Orthogonality of matrices and some distance problems, Linear Algebra Appl. 287 (1999), no. 1-3, 77-85.

[3] T. Bhattacharyya, P. Grover, Characterization of Birkhoff-James orthogonality, J. Math. Anal. Appl. 407 (2013), 350-358.

[4] G. BIR KhofF, Orthogonality in linear metric spaces, Duke Math. J. 1 (1935), 169-172.

[5] S. S. Dragomir, Semi-Inner Products and Applications, Nova Science Publishers, Inc., Hauppauge, NY, 2004.

[6] R. C. JAMEs, Orthogonality and linear functionals in normed linear spaces, Trans. Amer. Math. Soc., 61 (1947), 265-292.

[7] I. SINGER, Best Approximation in Normed Linear Spaces by Elements of Linear Subspaces, SpringerVerlag, Berlin, Heidelberg, New York, 1970. 